\author{
Military Technical College \\ Kobry El-Kobbah, \\ Cairo, Egypt.
}

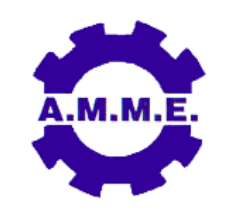

$17^{\text {th }}$ International Conference on Applied Mechanics and Mechanical Engineering.

\title{
DYNAMIC STRESS INTENSITY FACTORS FOR AN INTERFACIAL CRACK NEAR A NON-CIRCULAR CAVITY IN PIEZOELECTRIC BI-MATERIALS
}

\author{
A.H.M. Hassan ${ }^{1}$ and T.S. Song ${ }^{2}$
}

\begin{abstract}
A theoretical analysis is followed to calculate the dynamic stress intensity factors (DSIFs) due to existence of an interfacial crack near the right edge of a non-circular cavity, in transversely isotropic piezoelectric bi-materials. The model is subjected to dynamic incident anti-plane shearing (SH-wave). Green's functions are constructed based on complex variable and conformal mapping methods. DSIFs at the crack inner and outer tips are obtained by conjunction and cracks-deviation techniques. The boundary value problems are solved by applying the orthogonal function expansion technique. Based on FORTRAN language program, numerical calculations with an elliptic cavity are provided for different elliptic axial length ratios, different wave numbers and different piezoelectric parameters. For calibration, a comparison is accomplished between the present model and similar model with a crack emerging from a circular cavity edge. Calculating results showed the influences on DSIFs and how affected the efficiency of piezoelectric devices and materials.
\end{abstract}

\section{KEY WORDS}

Dynamic stress intensity factors (DSIFs), Interfacial crack, Piezoelectric bi-materials, Complex variable and conformal mapping methods, Non-circular cavity.

1 Egyptian Armed Forces.

2 Prof. Dr. in College of Aerospace and Civil Engineering, Harbin Engineering University, Harbin, China. 


\section{INTRODUCTION}

Piezoelectric smart materials are utilized widely in modern science technology. The electro-mechanical coupling response of these materials helps to establish plentiful styles of devices such as sensors, actuators and power supplies. However, faults and brittleness are occurring during manufacturing, polling process and service procedures due to materials stiffness and brittleness nature. That led researchers to investigate the defects behaviors and influences, and how they harm the efficiency of those devices.

At the last few years, the defects existed in piezoelectric materials have been analyzed in various arrangements and loading conditions. For example, Wu [1] developed an effective method to investigate the elastic field and the electric field of a crack in a confocal elliptic piezoelectric inhomogeneity embedded in an infinite piezoelectric medium, using the conformal mapping and the theorem of analytic continuation. The matrix is subjected to the remote anti-plane shear and in-plane electric field. Chen et. al. [2] investigated the dynamic stress concentration and scattering of SH-waves by bi-material structures that possess an interface elliptic cavity, by using the complex function method to construct Green's function. Liu and Wang [3] analyzed the electroelastic interaction of a screw dislocation and a notch in a piezoelectric bi-material using the conformal mapping and the image-dislocation approach. Liu and Lin [4] contracted a suitable Green's function for scattering of SH-waves and dynamic stress concentration by an interacting interface crack and a circular cavity near bi-material interface, for an elastic half space with a circular cavity impacted by an out-plane harmonic line source loading at the horizontal surface.

Liu and Chen [5] investigated the problem of SH-wave scattering by radial cracks of any limited length along the radius originating at the boundary of an elliptical hole, using complex function and Green's function methods. Chen et. al. [6] presented a novel efficient procedure to analyze the elliptical inhomogeneity problem in piezoelectric materials under electromechanical loadings, which include a point force and a point charge or a far-field anti-plane shear and in-plane electric field, using Green's function and conformal mapping. Sasaki et. al. [7] performed a twodimensional electro-elastic analysis on transversely isotropic piezoelectric materials containing an arbitrarily shaped boundary under out-of-plane mechanical and in-plane electrical loads at infinity, using the complex variable function method and the conformal mapping technique. Guo et. al. [8] studied the fracture problem of a semiinfinite crack in a piezoelectric strip using complex variable function method and conformal mapping technique, under the anti-plane shear stress and the in-plane electric load.

In transversely isotropic piezoelectric bi-materials, the objective is to evaluate theoretically the dynamic stress intensity factors (DSIFs) due to existence of an interfacial crack near the right edge of a non-circular cavity. A previous case was studied due to the existence of two symmetrically interfacial cracks near a non-circular cavity [9]. This model is subjected to dynamic incident anti-plane shearing (SH-wave). Based on complex variable and conformal mapping methods, Green's functions are constructed and the DSIFs at the crack inner and outer tips are obtained by conjunction and cracks-deviation techniques. The boundary value problems are solved by applying the orthogonal function expansion technique. Numerical examples with an elliptic 
cavity are provided for different elliptic axial length ratios, different wave numbers and different piezoelectric parameters, based on FORTRAN language program. Calculating results clarified the influences of the physical parameters, the structural geometry and the wave frequencies on the dimensionless DSIFs and how affected the efficiency of piezoelectric devices and materials.

\section{GOVERNING EQUATIONS}

Consider two transversely isotropic semi-infinite piezoelectric media $P M_{I}$ and $P M_{I I}$, located in the $X Y$-plane and the positive $Z$ axis is the polling direction. There exists an interfacial crack near the right edge of a non-circular cavity, subjected to dynamic SHwave as shown in Fig.1. The crack length is $A$ and the distance between the cavity edge and the crack inner tip is $B$. The positions of the crack's inner and outer tips are $\eta_{1}$ and $\eta_{2}$, respectively. The general expression of the time-harmonic and the twodimensional field are $[9,10,11]$ :

$$
F^{*}(X . Y . t)=F(X . Y) e^{-i \omega t}
$$

where $F^{*}$ is the desired field variable and $\omega$ is the incident wave frequency. For the sake of convenience, the exponential $e^{-i \omega t}$ is omitted [12]. In the absence of body forces and free charges, the governing equations of linear piezoelectricity under the SH-wave effect are $[9,10,11]$ :

$$
c_{44} \nabla^{2} w+e_{15} \nabla^{2} \emptyset+\rho \omega^{2} w=0 . \quad e_{15} \nabla^{2} w-\kappa_{11} \nabla^{2} \emptyset=0 .
$$

where $c_{44}, e_{15}$ and $\kappa_{11}$ are shear elastic modulus, piezoelectric constant and dielectric constant of piezoelectric medium, respectively; while $w, \emptyset$ and $\rho$ are out-of-plane displacement, electric potential and mass density of the medium, respectively. Introducing complex variables $X+i Y=\omega(\eta), X-i Y=\overline{\omega(\eta)}$, the external field of a non-circular cavity in the $X Y$-plane can be transformed into one of a unit circle in $\eta$ plane, based on conformal mapping method, if only $\omega^{\prime}(\eta) \neq 0$ in the mapping domain $[9,12]$. The plane expressed by column coordinate system $(r . \theta)$ centered at the origin$o$, where $\eta=r e^{i \theta}$ and $R_{0}=1$ is the circular cavity radius as shown in Fig.1. So, the anti-plane shear stresses $\left(\tau_{r z}\right.$ and $\left.\tau_{\theta z}\right)$ and the in-plane electric displacements $\left(D_{r}\right.$ and $\left.D_{\theta}\right)$ for a piezoelectric solid, can be expressed by:

$$
\begin{aligned}
& \tau_{r z}=\frac{c_{44}}{\left|\omega^{\prime}(\eta)\right|}\left(\frac{\partial w}{\partial \eta} e^{i \theta}+\frac{\partial w}{\partial \bar{\eta}} e^{-i \theta}\right)+\frac{e_{15}}{\left|\omega^{\prime}(\eta)\right|}\left(\frac{\partial \emptyset}{\partial \eta} e^{i \theta}+\frac{\partial \emptyset}{\partial \bar{\eta}} e^{-i \theta}\right) . \\
& \tau_{\theta z}=\frac{i c_{44}}{\left|\omega^{\prime}(\eta)\right|}\left(\frac{\partial w}{\partial \eta} e^{i \theta}-\frac{\partial w}{\partial \bar{\eta}} e^{-i \theta}\right)+\frac{i e_{15}}{\left|\omega^{\prime}(\eta)\right|}\left(\frac{\partial \emptyset}{\partial \eta} e^{i \theta}-\frac{\partial \emptyset}{\partial \bar{\eta}} e^{-i \theta}\right) . \\
& D_{r}=\frac{e_{15}}{\left|\omega^{\prime}(\eta)\right|}\left(\frac{\partial w}{\partial \eta} e^{i \theta}+\frac{\partial w}{\partial \bar{\eta}} e^{-i \theta}\right)-\frac{\kappa_{11}}{\left|\omega^{\prime}(\eta)\right|}\left(\frac{\partial \emptyset}{\partial \eta} e^{i \theta}+\frac{\partial \emptyset}{\partial \bar{\eta}} e^{-i \theta}\right) . \\
& D_{\theta}=\frac{i e_{15}}{\left|\omega^{\prime}(\eta)\right|}\left(\frac{\partial w}{\partial \eta} e^{i \theta}-\frac{\partial w}{\partial \bar{\eta}} e^{-i \theta}\right)-\frac{i \kappa_{11}}{\left|\omega^{\prime}(\eta)\right|}\left(\frac{\partial \emptyset}{\partial \eta} e^{i \theta}-\frac{\partial \emptyset}{\partial \bar{\eta}} e^{-i \theta}\right) .
\end{aligned}
$$

Also, Eq.2 can be simplified further as:

$$
\frac{\partial^{2} w}{\partial \eta \partial \bar{\eta}}=\left(\frac{i k}{2}\right)^{2} \omega^{\prime}(\eta) \overline{\omega^{\prime}(\eta)} \omega(\eta \cdot \bar{\eta}) . \quad \frac{\partial^{2} f}{\partial \eta \partial \bar{\eta}}=0 .
$$


where $k=\omega \sqrt{\rho / c^{*}}$ is the wave number, while $c^{*}=c_{44}(1+\lambda)$ is the effective piezoelectric stiffness and $\lambda=e_{15}{ }^{2} / c_{44} \kappa_{11}$ is the dimensionless piezoelectric parameter of the medium. The electric potential can be defined by:

$$
\varnothing=\frac{e_{15}}{\kappa_{11}} w+f
$$

\section{GREEN'S FUNCTIONS AND BOUNDARY VALUE PROBLEMS}

Under dynamic incident SH-wave at an arbitrary point $\eta_{0}$ with angle $\theta_{0}$ on the horizontal interface, the fundamental solutions on Green's functions of elastic displacement $G_{w}$ and electric potential $G_{\emptyset}$ for a semi-infinite piezoelectric medium can be constructed as [2,5]:

$$
\begin{aligned}
& \left.\left[\frac{\omega(\eta)}{|\omega(\eta)|}\right]^{-n}\right\} . \\
& G_{\varnothing}=\frac{e_{15}}{\kappa_{11}} G_{w}+\frac{i}{2 c^{*}} H_{0}^{(1)}\left(k\left|\omega(\eta)-\omega\left(\eta_{0}\right)\right|\right)+\sum_{n=0}^{\infty} A_{n} H_{n}^{(1)}(k|\omega(\eta)|) \cdot\left\{\left[\frac{\omega(\eta)}{|\omega(\eta)|}\right]^{n}+\right. \\
&
\end{aligned}
$$

where $H_{n}^{(1)}$ is Hankel function of the first kind. Green's function in the notch $G_{\emptyset}^{c}$ can be defined by:

$$
G_{\varnothing}^{c}=\frac{i}{2 c^{*}}\left[D_{0}+\sum_{n=1}^{\infty}\left(D_{n} \eta^{n}+E_{n} \bar{\eta}^{n}\right)\right] .
$$

Consider that the dynamic incident SH-wave directed with an angle $\alpha_{0}$ in medium $P M_{I}$. The interfacial crack is existed near the right edge of the circular cavity in the equivalent mapping plane as shown in Fig.1. Superscripts $I, I I$ and $c$ are used to express variables in $P M_{I}, P M_{I I}$ and the cavity respectively. So, the boundary continuity conditions across the medium cavity interface, which assumed to be traction free and electrically permeable, should be in the following forms $[12,13]$ :

$$
\left\{D_{r}^{I}=D_{r}^{c} . \quad D_{r}^{I I}=D_{r}^{c} . \quad G_{\varnothing}^{I}=G_{\emptyset}^{c} . \quad G_{\varnothing}^{I I}=G_{\varnothing}^{c} . \quad \tau_{r z}^{I}=0 . \quad \tau_{r z}^{I I}=0\right\} . \quad|\eta| \in 1 .
$$

Unknown coefficients $A_{n} \cdot B_{n} \cdot C_{n} \cdot D_{n} \cdot E_{n}$ and $D_{0}$ in Green's functions can be calculated by applying the boundary continuity conditions above with the orthogonal function expansion technique and the following relations about Hankel function [2,9,12]:

$$
\begin{aligned}
& \frac{\partial}{\partial \eta}\left[H_{n}^{(1)}(k|\omega(\eta)|)\left\{\frac{\omega(\eta)}{|\omega(\eta)|}\right\}^{n}\right]=\frac{k}{2} H_{n-1}^{(1)}(k|\omega(\eta)|)\left\{\frac{\omega(\eta)}{|\omega(\eta)|}\right\}^{n-1} \omega^{\prime}(\eta) . \\
& \frac{\partial}{\partial \bar{\eta}}\left[H_{n}^{(1)}(k|\omega(\eta)|)\left\{\frac{\omega(\eta)}{|\omega(\eta)|}\right\}^{n}\right]=-\frac{k}{2} H_{n+1}^{(1)}(k|\omega(\eta)|)\left\{\frac{\omega(\eta)}{|\omega(\eta)|}\right\}^{n+1} \overline{\omega^{\prime}(\eta)} . \\
& \frac{\partial}{\partial \eta}\left[H_{n}^{(1)}(k|\omega(\eta)|)\left\{\frac{\omega(\eta)}{|\omega(\eta)|}\right\}^{-n}\right]=-\frac{k}{2} H_{n+1}^{(1)}(k|\omega(\eta)|)\left\{\frac{\omega(\eta)}{|\omega(\eta)|}\right\}^{-n-1} \omega^{\prime}(\eta) . \\
& \frac{\partial}{\partial \bar{\eta}}\left[H_{n}^{(1)}(k|\omega(\eta)|)\left\{\frac{\omega(\eta)}{|\omega(\eta)|}\right\}^{-n}\right]=\frac{k}{2} H_{n-1}^{(1)}(k|\omega(\eta)|)\left\{\frac{\omega(\eta)}{|\omega(\eta)|}\right\}^{-n+1} \overline{\omega^{\prime}(\eta)} .
\end{aligned}
$$

So, the next relations can be obtained: 


$$
\begin{aligned}
& D_{n}=-\frac{\kappa_{11}}{\kappa_{0}} C_{n} . \quad E_{n}=-\frac{\kappa_{11}}{\kappa_{0}} B_{n} . \\
& \frac{e_{15}}{\kappa_{11}} \sum_{n=0}^{\infty} A_{n} H_{n}^{(1)}(k|\omega(\eta)|) \cdot\left\{\left[\frac{\omega(\eta)}{|\omega(\eta)|}\right]^{n}+\left[\frac{\omega(\eta)}{|\omega(\eta)|}\right]^{-n}\right\}-D_{0} \\
& +\frac{i}{2 c^{*}}\left(1+\frac{\kappa_{11}}{\kappa_{0}}\right) \sum_{n=1}^{\infty}\left(B_{n} e^{-i n \theta}+C_{n} e^{i n \theta}\right)=-\frac{e_{15} i}{2 \kappa_{11} c^{*}} \cdot H_{0}^{(1)}\left(k\left|\omega(\eta)-\omega\left(\eta_{0}\right)\right|\right) . \\
& k\left[\sum_{n=0}^{\infty} A_{n} H_{n-1}^{(1)}(k|\omega(\eta)|)\left\{\frac{\omega(\eta)}{|\omega(\eta)|}\right\}^{n-1}-\sum_{n=0}^{\infty} A_{n} H_{n+1}^{(1)}(k|\omega(\eta)|)\left\{\frac{\omega(\eta)}{|\omega(\eta)|}\right\}^{-n-1}\right] \omega^{\prime}(\eta) e^{i \theta} \\
& -k\left[\sum_{n=0}^{\infty} A_{n} H_{n+1}^{(1)}(k|\omega(\eta)|)\left\{\frac{\omega(\eta)}{|\omega(\eta)|}\right\}^{n+1}-\sum_{n=0}^{\infty} A_{n} H_{n-1}^{(1)}(k|\omega(\eta)|)\left\{\frac{\omega(\eta)}{|\omega(\eta)|}\right\}^{-n+1}\right] \frac{\omega^{\prime}(\eta)}{e^{-i \theta}} \\
& -\frac{e_{15} n i}{c^{* 2}} \sum_{n=1}^{\infty}\left(B_{n} e^{-i n \theta}+C_{n} e^{i n \theta}\right)=-\frac{k i}{2 c^{*}} H_{-1}^{(1)}\left(k\left|\omega(\eta)-\omega\left(\eta_{0}\right)\right|\right) \cdot\left\{\frac{\left(\eta_{0}\right)}{\left|\omega(\eta)-\omega\left(\eta_{0}\right)\right|}\right\} \omega^{\prime}(\eta) e^{i \theta} \\
& -\frac{k i}{2 c^{*}} H_{-1}^{(1)}\left(k\left|\omega(\eta)-\omega\left(\eta_{0}\right)\right|\right) \cdot\left\{\frac{\omega(\eta)-\omega\left(\eta_{0}\right)}{\left|\omega(\eta)-\omega\left(\eta_{0}\right)\right|}\right\} \overline{\omega^{\prime}(\eta)} e^{-i \theta} .
\end{aligned}
$$

where $\kappa_{0}$ is the permittivity dielectric constant of gas or vacuum inside cavity. Multiplying both sides of Eq.13 and Eq.14 by factor $e^{-i m \theta}, m=0 . \pm 1 . \pm 2 \ldots$ and integrating them from 0 to $2 \pi$ about $\theta$, the final expressions have the form of infinite linear algebraic system of equations about the unknown coefficients $[9,12]$ :

$$
\sum_{n=0}^{\infty} A_{n} p_{l n}+\sum_{n=1}^{\infty} B_{n} q_{l n}+\sum_{n=1}^{\infty} C_{n} s_{l n}+D_{0} \beta_{l}=\varepsilon_{l} . \quad l=1.2 .
$$

in which

$$
\begin{aligned}
& p_{1 n}=\frac{e_{15}}{2 \pi \kappa_{11}} \int_{0}^{2 \pi} H_{n}^{(1)}(k|\omega(\eta)|) \cdot\left\{\left[\frac{\omega(\eta)}{|\omega(\eta)|}\right]^{n}+\left[\frac{\omega(\eta)}{|\omega(\eta)|}\right]^{-n}\right\} e^{-i m \theta} d \theta . \\
& p_{2 n}=\frac{k}{2 \pi} \int_{0}^{2 \pi}\left[H_{n-1}^{(1)}(k|\omega(\eta)|)\left\{\left[\frac{\omega(\eta)}{|\omega(\eta)|}\right]^{n-1}\right\}-H_{n+1}^{(1)}(k|\omega(\eta)|) \cdot\left\{\left[\frac{\omega(\eta)}{|\omega(\eta)|}\right]^{-n-1}\right\}\right] \omega^{\prime}(\eta) e^{i \theta} e^{-i m \theta} d \theta \\
& \quad-\frac{k}{2 \pi} \int_{0}^{2 \pi}\left[H_{n+1}^{(1)}(k|\omega(\eta)|)\left\{\left[\frac{\omega(\eta)}{|\omega(\eta)|}\right]^{n+1}\right\}-H_{n-1}^{(1)}(k|\omega(\eta)|) \cdot\left\{\left[\frac{\omega(\eta)}{|\omega(\eta)|}\right]^{-n+1}\right\}\right] \overline{\omega^{\prime}(\eta)} e^{-i \theta} e^{-i m \theta} d \theta . \\
& q_{1 n}=\left(1+\frac{\kappa_{11}}{\kappa_{0}}\right) \frac{i}{4 \pi c^{*}} \int_{0}^{2 \pi} e^{-i n \theta} e^{-i m \theta} d \theta . \quad \& \quad q_{2 n}=-\frac{e_{15} n i}{2 \pi c^{* 2}} \int_{0}^{2 \pi} e^{-i n \theta} e^{-i m \theta} d \theta . \\
& s_{1 n}=\left(1+\frac{\kappa_{11}}{\kappa_{0}}\right) \frac{i}{4 \pi c^{*}} \int_{0}^{2 \pi} e^{i n \theta} e^{-i m \theta} d \theta . \quad \& \quad s_{2 n}=-\frac{e_{15} n i}{2 \pi c^{* 2}} \int_{0}^{2 \pi} e^{i n \theta} e^{-i m \theta} d \theta . \\
& \beta_{1}=-\frac{1}{2 \pi} \int_{0}^{2 \pi} e^{-i m \theta} d \theta . \quad \& \quad \beta_{2}=0 . \\
& \varepsilon_{1}=-\frac{e_{15} i}{4 \pi c^{*} \kappa_{11}} \int_{0}^{2 \pi} H_{0}^{(1)}\left(k\left|\omega(\eta)-\omega\left(\eta_{0}\right)\right|\right) e^{-i m \theta} d \theta . \\
& \varepsilon_{2}=-\frac{k i}{4 \pi c^{*}} \int_{0}^{2 \pi} H_{-1}^{(1)}\left(k\left|\omega(\eta)-\omega\left(\eta_{0}\right)\right|\right) \cdot\left\{\frac{\overline{\omega(\eta)}-\overline{\omega\left(\eta_{0}\right)}}{\left|\omega(\eta)-\omega\left(\eta_{0}\right)\right|}\right\} \omega^{\prime}(\eta) e^{i \theta} e^{-i m \theta} d \theta \\
&-\frac{k i}{4 \pi c^{*}} \int_{0}^{2 \pi} H_{-1}^{(1)}\left(k\left|\omega(\eta)-\omega\left(\eta_{0}\right)\right|\right) \cdot\left\{\frac{\omega(\eta)-\omega\left(\eta_{0}\right)}{\left|\omega(\eta)-\omega\left(\eta_{0}\right)\right|}\right\} \overline{\omega^{\prime}(\eta)} e^{-i \theta} e^{-i m \theta} d \theta .
\end{aligned}
$$

By truncating, the polynomials in Eq. 15 to the $N$ th term, and meanwhile by taking $n=$ $0 . \pm 1 . \pm 2 \ldots \pm N$, expressions can be reduced to a finite linear algebraic system of equations with $(4 N+2)$ equations and $(4 N+2)$ unknown coefficients. By solving the reduced expressions, the unknown coefficients can be calculated. The practical calculating indicates that satisfied calculations can be obtained only by taking $N=8$.

\section{DYNAMIC ELECTRO-ELASTIC FIELDS}

Different impedances at the interface joining the piezoelectric bi-materials caused 
scattering phenomena. The dynamic electro-elastic and scattering fields can be given as follows $[2,5]$ :

(1) Incident elastic displacement and electric potential fields in $P M_{I}$ :

$$
w^{(i)}=w_{0} \exp \left\{-\frac{i k_{I}}{2}\left[\omega(\eta) e^{i \alpha_{0}}+\overline{\omega(\eta)} e^{-i \alpha_{0}}\right]\right\} . \quad \emptyset^{(i)}=\frac{e_{15}^{I}}{\kappa_{11}^{I}} w^{(i)} .
$$

where $w_{0}$ is the magnitude of incident displacement wave.

(2) Reflecting and scattering electro-elastic fields in $P M_{I}$ :

$$
\begin{aligned}
& w^{(r)}=w_{1} \exp \left\{-\frac{i k_{I}}{2}\left[\omega(\eta) e^{i \alpha_{1}}+\overline{\omega(\eta)} e^{-i \alpha_{1}}\right]\right\} . \quad \phi^{(r)}=\frac{e_{15}^{I}}{\kappa_{11}^{I}} w^{(r)} . \\
& w^{(s)}=\sum_{n=-\infty}^{\infty} A_{n}^{(i s)} H_{n}^{(1)}\left(k_{I}|\omega(\eta)|\right)\left\{\frac{\omega(\eta)}{|\omega(\eta)|}\right\}^{n}+\sum_{n=-\infty}^{\infty} A_{n}^{(r s)} H_{n}^{(1)}\left(k_{I}|\omega(\eta)|\right)\left\{\frac{\omega(\eta)}{|\omega(\eta)|}\right\}^{n} . \\
& \emptyset^{(s)}=\frac{e_{15}^{I}}{\kappa_{11}^{I}} w^{(s)}+\frac{i}{2 c_{I}^{*}}\left[\sum_{n=1}^{\infty}\left(B_{n}^{(i s)} \eta^{-n}+C_{n}^{(i s)} \bar{\eta}^{-n}\right)+\sum_{n=1}^{\infty}\left(B_{n}^{(r s)} \eta^{-n}+\right.\right. \\
& \left.\left.C_{n}^{(r s)} \bar{\eta}^{-n}\right)\right]
\end{aligned}
$$

where $w_{1}$ and $\alpha_{1}$ are the magnitude and angle of reflecting displacement wave, respectively.

(3) Refracting and scattering electro-elastic fields in $P M_{I I}$ :

$$
\begin{aligned}
& w^{(f)}=w_{2} \exp \left\{-\frac{i k_{I I}}{2}\left[\omega(\eta) e^{i \alpha_{2}}+\overline{\omega(\eta)} e^{-i \alpha_{2}}\right]\right\} . \quad \emptyset^{(f)}=\frac{e_{15}^{I I}}{\kappa_{11}^{I I}} w^{(f)} . \\
& w^{(f s)}=\sum_{n=-\infty}^{\infty} A_{n}^{(f s)} H_{n}^{(1)}\left(k_{I I}|\omega(\eta)|\right)\left\{\frac{\omega(\eta)}{|\omega(\eta)|}\right\}^{n} . \\
& \emptyset^{(f s)}=\frac{e_{15}^{I I}}{\kappa_{11}^{I I}} w^{(f s)}+\frac{i}{2 c_{I I}^{*}}\left[\sum_{n=1}^{\infty}\left(B_{n}^{(f s)} \eta^{-n}+C_{n}^{(f s)} \bar{\eta}^{-n}\right)\right] .
\end{aligned}
$$

where $w_{2}$ and $\alpha_{2}$ are the magnitude and angle of refracting displacement wave, respectively.

(4) For electric potential inside the circular cavity, the cavity is assumed to be vacuum or filled with homogeneous gas of dielectric constant $\kappa_{0}$, and free of forces and surface charges:

$$
\begin{aligned}
& \emptyset^{c(i s)}=\frac{i}{2 c_{I}^{*}}\left[D_{0}^{(i s)}+\sum_{n=1}^{\infty}\left(D_{n}^{(i s)} \eta^{n}+E_{n}^{(i s)} \bar{\eta}^{n}\right)\right] . \\
& \emptyset^{c(r s)}=\frac{i}{2 c_{I}^{*}}\left[D_{0}^{(r s)}+\sum_{n=1}^{\infty}\left(D_{n}^{(r s)} \eta^{n}+E_{n}^{(r s)} \bar{\eta}^{n}\right)\right] . \\
& \emptyset^{c(f s)}=\frac{i}{2 c_{I I}^{*}}\left[D_{0}^{(f s)}+\sum_{n=1}^{\infty}\left(D_{n}^{(f s)} \eta^{n}+E_{n}^{(f s)} \bar{\eta}^{n}\right)\right] .
\end{aligned}
$$

The total electro-elastic fields for the two media $P M_{I}$ and $P M_{I I}$ respectively:

$$
w^{I}=w^{(i)}+w^{(r)}+w^{(s)} . \quad \emptyset^{I}=\emptyset^{(i)}+\emptyset^{(r)}+\emptyset^{(s)} .
$$




$$
w^{I I}=w^{(f)}+w^{(f s)} . \quad \emptyset^{I I}=\emptyset^{(f)}+\emptyset^{(f s)} .
$$

All unknown coefficients for incident, reflecting and refracting fields can also be calculated with the help of the orthogonal function expansion technique by applying the boundary continuity conditions across the medium cavity interface, which should be in the following forms:

$$
\left\{D_{r}^{I}=D_{r}^{c} . \quad D_{r}^{I I}=D_{r}^{c} . \quad \emptyset^{I}=\emptyset^{c} . \quad \emptyset^{I I}=\emptyset^{c} . \quad \tau_{r z}^{I}=0 . \quad \tau_{r z}^{I I}=0\right\} . \quad|\eta| \in 1 .
$$

For the incident unknown coefficients $A_{n}^{(i s)} \cdot B_{n}^{(i s)} \cdot C_{n}^{(i s)} \cdot D_{n}^{(i s)} \cdot E_{n}^{(i s)} \cdot D_{0}^{(i s)}$, the next relations can be obtained:

$$
\begin{aligned}
& D_{n}^{(i s)}=-\frac{\kappa_{11}^{I}}{\kappa_{0}} C_{n}^{(i s)} \cdot \quad E_{n}^{(i s)}=-\frac{\kappa_{11}^{I}}{\kappa_{0}} B_{n}^{(i s)} . \\
& \frac{e_{15}^{I}}{\kappa_{11}^{I}} \sum_{n=-\infty}^{\infty} A_{n}^{(i s)} H_{n}^{(1)}\left(k_{I}|\omega(\eta)|\right) \cdot\left\{\left[\frac{\omega(\eta)}{|\omega(\eta)|}\right]^{n}\right\}+\left(1+\frac{\kappa_{11}^{I}}{\kappa_{0}}\right) \frac{i}{2 c_{I}^{*}} \sum_{n=1}^{\infty}\left(B_{n}^{(i s)} e^{-i n \theta}+C_{n}^{(i s)} e^{i n \theta}\right) \\
& -D_{0}^{(i s)}=-\frac{e_{15}^{I}}{\kappa_{11}^{I}} w_{0} \exp \left\{-\frac{i k_{I}}{2}\left[\omega(\eta) e^{i \alpha_{0}}+\overline{\omega(\eta)} e^{-i \alpha_{0}}\right]\right\} \\
& k_{I} \sum_{n=-\infty}^{\infty} A_{n}^{(i s)} H_{n-1}^{(1)}\left(k_{I}|\omega(\eta)|\right)\left\{\frac{\omega(\eta)}{|\omega(\eta)|}\right\}^{n-1} \omega^{\prime}(\eta) e^{i \theta} \\
& -k_{I} \sum_{n=-\infty}^{\infty} A_{n}^{(i s)} H_{n+1}^{(1)}\left(k_{I}|\omega(\eta)|\right)\left\{\frac{\omega(\eta)}{|\omega(\eta)|}\right\}^{n+1} \overline{\omega^{\prime}(\eta)} e^{-i \theta} \\
& -\frac{e_{15}^{I} n i}{c_{I}^{* 2}} \sum_{n=1}^{\infty}\left(B_{n}^{(i s)} e^{-i n \theta}+C_{n}^{(i s)} e^{i n \theta}\right)= \\
& i k_{I} w_{0} \exp \left\{-\frac{i k_{I}}{2}\left[\omega(\eta) e^{i \alpha_{0}}+\overline{\omega(\eta)} e^{-i \alpha_{0}}\right]\right\} \cdot\left\{e^{i \alpha_{0}} \omega^{\prime}(\eta) e^{i \theta}+e^{-i \alpha_{0}} \overline{\omega^{\prime}(\eta)} e^{-i \theta}\right\}
\end{aligned}
$$

The infinite linear algebraic systems of equations are as follows:

$$
\sum_{n=-\infty}^{\infty} A_{n}^{(i s)} p_{l n}+\sum_{n=1}^{\infty} B_{n}^{(i s)} q_{l n}+\sum_{n=1}^{\infty} C_{n}^{(i s)} s_{l n}+D_{0}^{(i s)} \beta_{l}=\varepsilon_{l} . \quad l=1.2 .
$$

in which

$$
\begin{aligned}
p_{1 n} & =\frac{e_{15}^{I}}{2 \pi \kappa_{11}^{I}} \int_{0}^{2 \pi} H_{n}^{(1)}\left(k_{I}|\omega(\eta)|\right) \cdot\left\{\left[\frac{\omega(\eta)}{|\omega(\eta)|}\right]^{n}\right\} e^{-i m \theta} d \theta . \\
p_{2 n} & =\frac{k_{I}}{2 \pi} \int_{0}^{2 \pi} H_{n-1}^{(1)}\left(k_{I}|\omega(\eta)|\right)\left\{\frac{\omega(\eta)}{|\omega(\eta)|}\right\}^{n-1} \omega^{\prime}(\eta) e^{i \theta} e^{-i m \theta} d \theta \\
& -\frac{k_{I}}{2 \pi} \int_{0}^{2 \pi} H_{n+1}^{(1)}\left(k_{I}|\omega(\eta)|\right)\left\{\frac{\omega(\eta)}{|\omega(\eta)|}\right\}^{n+1} \overline{\omega^{\prime}(\eta)} e^{-i \theta} e^{-i m \theta} d \theta . \\
q_{1 n} & =\left(1+\frac{\kappa_{11}^{I}}{\kappa_{0}}\right) \frac{i}{4 \pi c_{I}^{*}} \int_{0}^{2 \pi} e^{-i n \theta} e^{-i m \theta} d \theta . \quad \& \quad q_{2 n}=-\frac{e_{15}^{I} n i}{2 \pi c_{I}^{* 2}} \int_{0}^{2 \pi} e^{-i n \theta} e^{-i m \theta} d \theta . \\
s_{1 n} & =\left(1+\frac{\kappa_{11}^{I}}{\kappa_{0}}\right) \frac{i}{4 \pi c_{I}^{*}} \int_{0}^{2 \pi} e^{i n \theta} e^{-i m \theta} d \theta . \quad \& \quad s_{2 n}=-\frac{e_{15}^{I} n i}{2 \pi c_{I}^{* 2}} \int_{0}^{2 \pi} e^{i n \theta} e^{-i m \theta} d \theta . \\
\beta_{1}= & -\frac{1}{2 \pi} \int_{0}^{2 \pi} e^{-i m \theta} d \theta . \quad \& \quad \beta_{2}=0 . \\
\varepsilon_{1}= & -\frac{e_{15}^{I} w_{0}}{2 \pi \kappa_{11}^{I}} \int_{0}^{2 \pi} \exp \left\{-\frac{i k_{I}}{2}\left[\omega(\eta) e^{i \alpha_{0}}+\overline{\omega(\eta)} e^{-i \alpha_{0}}\right]\right\} \cdot e^{-i m \theta} d \theta . \\
\varepsilon_{2}= & \frac{k_{I} w_{0} i}{2 \pi} \int_{0}^{2 \pi} \exp \left\{-\frac{i k_{I}}{2}\left[\omega(\eta) e^{i \alpha_{0}}+\overline{\omega(\eta)} e^{-i \alpha_{0}}\right]\right\} \cdot e^{i \alpha_{0}} \omega^{\prime}(\eta) e^{i \theta} e^{-i m \theta} d \theta \\
& +\frac{k_{I} w_{0} i}{2 \pi} \int_{0}^{2 \pi} \exp \left\{-\frac{i k_{I}}{2}\left[\omega(\eta) e^{i \alpha_{0}}+\overline{\omega(\eta)} e^{-i \alpha_{0}}\right]\right\} \cdot e^{-i \alpha_{0}} \overline{\omega^{\prime}(\eta)} e^{-i \theta} e^{-i m \theta} d \theta .
\end{aligned}
$$


The incident unknown coefficients can be calculated by truncating the polynomials in Eq.28 with the sequence described before in Eq.15. Also, the same procedures can be followed to calculate the unknown coefficients for reflecting field $A_{n}^{(r s)} \cdot B_{n}^{(r s)} \cdot C_{n}^{(r s)} \cdot D_{n}^{(r s)} \cdot E_{n}^{(r s)}$. $D_{0}^{(r s)}$ and the unknown coefficients for refracting field $A_{n}^{(f s)} \cdot B_{n}^{(f s)} \cdot C_{n}^{(f s)} \cdot D_{n}^{(f s)} \cdot E_{n}^{(f s)} \cdot D_{0}^{(f s)}$.

\section{INTEGRAL EQUATION AND DSIFs}

The conjunction and crack-deviation techniques are used to evaluate the integral equation and calculate DSIFs at the crack inner and outer tips. The piezoelectric bimaterials' conjunction is shown in Fig.2. The interface intervals in mapping plane can be defined as:

$$
\left\{\begin{array}{ll}
\Gamma_{1} \in\left[R_{0} \cdot R_{0}+B\right] \cdot \theta=0 ; & \Gamma_{2}>R_{0}+B+A \cdot \theta=0 \\
\Gamma_{3}>R_{0} \cdot \theta=\pi ; & C \in\left[R_{0}+B \cdot R_{0}+B+A\right] \cdot \theta=0 .
\end{array}\right\} .
$$

To apply the crack-deviation technique, two negative shear stresses $-\tau_{\theta z}^{I}$ and $-\tau_{\theta Z}^{I I}$ are estimated at the crack location. The continuity conditions of shear stresses excluding the areas of the crack and the cavity can be expressed by $[5,9,13]$ :

$$
\tau_{\theta Z}^{I} \cos \theta_{0}+f_{1}\left(r_{0} \cdot \theta_{0}\right)=\tau_{\theta Z}^{I I} \cos \theta_{0}+f_{2}\left(r_{0} \cdot \theta_{0}\right) \text {. at } \Gamma_{1} \cdot \Gamma_{2} \text { and } \Gamma_{3} \text {. }
$$

where $f_{1}\left(r_{0} \cdot \theta_{0}\right)$ and $f_{2}\left(r_{0} \cdot \theta_{0}\right)$ are two additional stresses applied at the well-bounded interfaces. Returning to Eq.3, it can be concluded that [9]:

$$
\tau_{\theta z}^{I}=\tau_{\theta z}^{(i)}+\tau_{\theta z}^{(r)}+\tau_{\theta z}^{(s)} . \quad \tau_{\theta z}^{I I}=\tau_{\theta z}^{(f)}+\tau_{\theta z}^{(f s)} .
$$

in which:

$$
\begin{aligned}
\tau_{\theta z}^{(i)}= & \frac{k_{I} c_{I}^{*} \omega_{0}}{2\left|\omega^{\prime}(\eta)\right|}\left[\exp \left\{-\frac{i k_{I}}{2}\left[\omega(\eta) e^{i \alpha_{0}}+\overline{\omega(\eta)} e^{-i \alpha_{0}}\right]\right\} \cdot\left\{e^{i \alpha_{0}} \omega^{\prime}(\eta) e^{i \theta}-e^{-i \alpha_{0}} \overline{\omega^{\prime}(\eta)} e^{-i \theta}\right\}\right] . \\
\tau_{\theta z}^{(r)}= & \frac{k_{I} c_{I}^{\prime} \omega_{1}}{2\left|\omega^{\prime}(\eta)\right|}\left[\exp \left\{-\frac{i k_{I}}{2}\left[\omega(\eta) e^{i \alpha_{1}}+\overline{\omega(\eta)} e^{-i \alpha_{1}}\right]\right\} \cdot\left\{e^{i \alpha_{1}} \omega^{\prime}(\eta) e^{i \theta}-e^{-i \alpha_{1}} \overline{\omega^{\prime}(\eta)} e^{-i \theta}\right\}\right] . \\
\tau_{\theta z}^{(s)}= & \frac{i k_{I} c_{I}^{*}}{2\left|\omega^{\prime}(\eta)\right|} \sum_{n=-\infty}^{\infty} A_{n}^{(i s)}\left[H_{n-1}^{(1)}\left(k_{I}|\omega(\eta)|\right)\left\{\frac{\omega(\eta)}{|\omega(\eta)|}\right\}^{n-1} \omega^{\prime}(\eta) e^{i \theta}\right. \\
& \left.+H_{n+1}^{(1)}\left(k_{I}|\omega(\eta)|\right)\left\{\frac{\omega(\eta)}{|\omega(\eta)|}\right\}^{n+1} \overline{\omega^{\prime}(\eta)} e^{-i \theta}\right]+\frac{e_{15}^{I}}{2 c_{I}^{*}\left|\omega^{\prime}(\eta)\right|} \sum_{n=1}^{\infty}\left(n B_{n}^{(i s)} e^{-i n \theta}-n C_{n}^{(i s)} e^{i n \theta}\right) \\
& +\frac{i k_{1} c_{I}^{*}}{2\left|\omega^{\prime}(\eta)\right|} \sum_{n=-\infty}^{\infty} A_{n}^{(r s)}\left[H_{n-1}^{(1)}\left(k_{I}|\omega(\eta)|\right)\left\{\frac{\omega(\eta)}{|\omega(\eta)|}\right\}^{n-1} \omega^{\prime}(\eta) e^{i \theta}\right. \\
& \left.+H_{n+1}^{(1)}\left(k_{I}|\omega(\eta)|\right)\left\{\frac{\omega(\eta)}{|\omega(\eta)|}\right\}^{n+1} \overline{\omega^{\prime}(\eta)} e^{-i \theta}\right]+\frac{e_{15}^{I}}{2 c_{I}^{*}\left|\omega^{\prime}(\eta)\right|} \sum_{n=1}^{\infty}\left(n B_{n}^{(r s)} e^{-i n \theta}-n C_{n}^{(r s)} e^{i n \theta}\right) . \\
\tau_{\theta z}^{(f)}= & \frac{k_{I I} c_{I I}^{*} w_{2}}{2\left|\omega^{\prime}(\eta)\right|}\left[\exp \left\{-\frac{i k_{I I}}{2}\left[\omega(\eta) e^{i \alpha_{2}}+\overline{\omega(\eta)} e^{-i \alpha_{2}}\right]\right\} \cdot\left\{e^{i \alpha_{2}} \omega^{\prime}(\eta) e^{i \theta}-e^{-i \alpha_{2}} \overline{\omega^{\prime}(\eta)} e^{-i \theta}\right\}\right] . \\
\tau_{\theta z}^{(f s)}= & \frac{i k_{I I} c_{I I}^{*}}{2\left|\omega^{\prime}(\eta)\right|} \sum_{n=-\infty}^{\infty} A_{n}^{(f s)}\left[H_{n-1}^{(1)}\left(k_{I I}|\omega(\eta)|\right)\left\{\frac{\omega(\eta)}{|\omega(\eta)|}\right\}^{n-1} \omega^{\prime}(\eta) e^{i \theta}\right. \\
& \left.+H_{n+1}^{(1)}\left(k_{I I}|\omega(\eta)|\right)\left\{\frac{\omega(\eta)}{|\omega(\eta)|}\right\}^{n+1} \overline{\omega^{\prime}(\eta)} e^{-i \theta}\right]+\frac{e_{15}^{I I}}{2 c_{I I}^{*}\left|\omega^{\prime}(\eta)\right|} \sum_{n=1}^{\infty}\left(n B_{n}^{(f s)} e^{-i n \theta}-n C_{n}^{(f s)} e^{i n \theta}\right) .
\end{aligned}
$$

The continuity conditions of elastic displacements can be expressed by $[9,13]$ : 


$$
w^{I}(r . \theta)+w^{\left(f_{1}\right)}(r . \theta)+w^{\left(C_{r} I\right)}(r . \theta)=w^{I I}(r . \theta)+w^{\left(f_{2}\right)}(r . \theta)+w^{\left(C_{r} I I\right)}(r . \theta) .
$$

where:

$$
\begin{aligned}
& w^{\left(f_{1}\right)}=\int_{\Gamma_{1} \& \Gamma_{2}} f_{1}\left(r_{0} .0\right) G_{w}^{I}\left(r . \theta ; r_{0} .0\right) d r_{0}+\int_{\Gamma_{3}} f_{1}\left(r_{0} . \pi\right) G_{w}^{I}\left(r . \theta ; r_{0} . \pi\right) d r_{0} . \\
& w^{\left(f_{2}\right)}=-\int_{\Gamma_{1} \& \Gamma_{2}} f_{2}\left(r_{0} .0\right) G_{w}^{I I}\left(r . \theta ; r_{0} .0\right) d r_{0}-\int_{\Gamma_{3}} f_{2}\left(r_{0} . \pi\right) G_{w}^{I I}\left(r . \theta ; r_{0} . \pi\right) d r_{0} . \\
& w^{\left(C_{r} I\right)}=-\int_{C} \tau_{\theta z}^{I}\left(r_{0} .0\right) G_{w}^{I}\left(r . \theta ; r_{0} .0\right) d r_{0} . \\
& w^{\left(C_{r} I I\right)}=\int_{C} \tau_{\theta z}^{I I}\left(r_{0} .0\right) G_{w}^{I I}\left(r . \theta ; r_{0} .0\right) d r_{0} .
\end{aligned}
$$

Gathering all the equations above, the integral equation can be calculated as follows [9,13]:

$$
\begin{aligned}
& \int_{\Gamma_{1} \& \Gamma_{2}} f_{1}\left(r_{0} .0\right)\left[G_{w}^{I}\left(r . \theta ; r_{0} .0\right)+G_{w}^{I I}\left(r . \theta ; r_{0} .0\right)\right] d r_{0} \\
& +\int_{\Gamma_{3}} f_{1}\left(r_{0} . \pi\right)\left[G_{w}^{I}\left(r . \theta ; r_{0} . \pi\right)+G_{w}^{I I}\left(r . \theta ; r_{0} . \pi\right)\right] d r_{0}=w^{(f s)}(r . \theta)-w^{(s)}(r . \theta) \\
& -\int_{\Gamma_{1} \& \Gamma_{2}}\left[\tau_{\theta z}^{I}\left(r_{0} .0\right)-\tau_{\theta z}^{I I}\left(r_{0} .0\right)\right] G_{w}^{I I}\left(r . \theta ; r_{0} .0\right) d r_{0} \\
& +\int_{\Gamma_{3}}\left[\tau_{\theta z}^{I}\left(r_{0} . \pi\right)-\tau_{\theta z}^{I I}\left(r_{0} . \pi\right)\right] G_{w}^{I I}\left(r . \theta ; r_{0} . \pi\right) d r_{0} \\
& +\int_{C} \tau_{\theta z}^{I}\left(r_{0} .0\right) G_{w}^{I}\left(r . \theta ; r_{0} .0\right) d r_{0}+\int_{C} \tau_{\theta z}^{I I}\left(r_{0} .0\right) G_{w}^{I I}\left(r . \theta ; r_{0} .0\right) d r_{0} . \quad \theta=0 . \pi .
\end{aligned}
$$

Finally, the dimensionless DSIFs $\left(k_{3}^{\sigma}\right)$ at $\eta_{1}$ and $\eta_{2}$ can be defined by:

$$
\begin{aligned}
& k_{3 \eta_{1}}^{\sigma}=\frac{1}{\tau_{0} Q}\left|\lim _{r_{0} \rightarrow \mathrm{R}_{0}+\mathrm{B}} f_{1}\left(r_{0} \cdot 0\right) \sqrt{2\left(r_{0}-R_{0}-\mathrm{B}\right.}\right| . \\
& k_{\eta_{\eta_{2}}}^{\sigma}=\frac{1}{\tau_{0} Q}\left|\lim _{r_{0} \rightarrow \mathrm{R}_{0}+\mathrm{B}+\mathrm{A}} f_{1}\left(r_{0} \cdot 0\right) \sqrt{2\left(r_{0}-R_{0}-\mathrm{B}-\mathrm{A}\right.}\right| .
\end{aligned}
$$

where the characteristic length $Q=\sqrt{A / 2}$ and $\tau_{0}=k_{I} w_{0} c_{I}^{*}$ refers to shear stress magnitude of the incident wave [5,13].

\section{NUMERICAL EXAMPLES AND DISCUSSIONS}

To examine the influences of different parameters on the DSIFs, some calculations are provided based on FORTRAN language program for Eq.34 and Eq.35, for a piezoelectric bi-materials media with an elliptic cavity. The conformal mapping function, which transforms the domain outside an ellipse in $X Y$-plane into one outside a unit circle in $\eta$-plane, is given by $[2,5,9]$ :

$$
X+i Y=\omega(\eta)=\frac{a+b}{2}\left(\eta+\frac{a-b}{a+b} \eta^{-1}\right)
$$

in which $a$ and $b$ represent the two semi-axis' lengths of the ellipse along the $X$-axis and the $Y$-axis, respectively.

First, for calibration of program efficiency, a comparison of DSIFs at $\eta_{2}$ is accomplished between the present model and the reference [13] model with crack emerging from the circular cavity edge as shown in Fig.3. The document model solved without using 
complex variable and conformal mapping methods. It can be noticed that the DSIFs of the two models increased progressively to reach values around 1.00. It is expectable and physically true that if letting $A / a \rightarrow \infty$, one can finds that DSIFs $\rightarrow 1[10,11,14]$.

The DSIFs of the present model gave lower values than the document model. The two curves did not coincide. So, using complex variable and conformal mapping methods in the current paper solution cause the wide change in DSIFs. Also, the distance $B$ is used very small $(B / a=0.001)$ but still existed as a separation between the crack and the cavity. It can be concluded that, depending on the medium's geometry and the sequence of calculations, the relation between the crack and the cavity is altered causing the changes occurred to the values of the DSIFs.

Variations of DSIFs at $\eta_{1}$ and $\eta_{2}$ for different semi-axis' percentages of the ellipse are shown in Fig. 4 and Fig.5 respectively. The outer tip gave the larger values of DSIFs than the inner tip (about 85\%-95\%). The peak of the curves occurred at almost the same $k_{I} a$ percentages for the two tips' curves. Then, the DSIFs continue to decrease rapidly with $k_{I} a$ increment. But when $b / a=1.5$, the decrement is occurred slowly. While the peak value of the curves decreased with the increment of semi-axis' percentages at $\eta_{1}$, it increased at $\eta_{2}$. So, the increment of the semi-axis' percentages led to the decrement of both the curve's peak at inner tip and the variation of DSIFs with $k_{I} a$. But, the increment led to increment of the curve's peak at outer tip.

Variations of DSIFs at $\eta_{1}$ and $\eta_{2}$ for different $A / a$ percentages are shown in Fig. 6 and Fig.7 respectively. The semi-axis' percentage is $b / a=0.8$. On both tips, the curve for percentage $A / a=0.5$ showed a very little increase of DSIFs with the increment of $k_{I} a$. While the percentage $A / a$ increased, the curve showed higher oscillation and decrease the DSIFs rapidly. On the outer tip, while the percentage $A / a$ increased, the peak value of the curve increased and the DSIFs decreased rapidly.

Variations of DSIFs at $\eta_{1}$ and $\eta_{2}$ with $A / a$ for different semi-axis' percentages of the ellipse are shown in Fig. 8 and Fig. 9 respectively. The outer tip gave the larger values of DSIFs than the inner tip. The peak of the curves occurred at almost the same $A / a$ percentages for the two tips' curves. In the inner tip, the peak value decrease with the increment of $b / a$ percentage. In the outer tip, the peak value decrement is a little small. The DSIFs curves continue to decrease rapidly with the $A / a$ increment. But, the values of DSIFs are larger for $b / a=1.5$ for the two tips' curves. So, the DSIFs decreased with the increment of the $A / a$, for different semi-axes' percentages and the semi-axes' percentage of the ellipse $b / a=1.5$ gave the smaller Peaks and the larger values of DSIFs for the two tips.

Variations of DSIFs at $\eta_{1}$ and $\eta_{2}$ with $A / a$ for different incident wave frequencies $\left(\lambda_{I}\right)$ are shown in Fig.10 and Fig.11 respectively. The curves at both tips are almost coincide while $\lambda_{I}$ changes. On both tips, the DSIFs curves continue to decrease rapidly while $A / a>2$. So, for different incident wave frequencies, the variations of DSIFs are not affected quite clear and they are decreased with the increment of $A / a$. It can be concluded that, the rise of the incident wave number harms the efficiency of piezoelectric devices and materials used, especially when the crack length is longer than twice the cavity's semi-axis $a$. 


\section{CONCLUSIONS}

In transversely isotropic piezoelectric bi-materials, a theoretical analysis is established to calculate the DSIFs due to existence of an interfacial crack near the right edge of a noncircular cavity. The model is subjected to dynamic SH-wave. Green's functions are constructed based on complex variable and conformal mapping methods. The DSIFs at the crack's inner and outer tips are obtained by conjunction and crack deviation techniques. Numerical calculations are provided with an elliptic cavity based on FORTRAN language program. Calculating results illustrated that, depending on the medium's geometry and the sequence of calculations, the relation between the crack and the cavity is altered causing the changes occurred to the values of the DSIFs. The outer tip gave the larger values of DSIFs than the inner tip in all cases of study. For the variations with the wave number, the increment of the semi-axes' percentages led to the decrement of both the curve's peak at inner tip and the variation of DSIFs. But, the increment led to the curve's peak increment at outer tip. For different incident wave frequencies, the variations of DSIFs are not affected quite clear. The DSIFs decreased with the increment of the crack length ratio for both the different semi-axes' percentages and the different incident wave frequencies. The rise of the incident wave number harms the efficiency of piezoelectric devices and materials used, especially when the crack length is longer than twice the cavity's semi-axis $a$.

\section{REFERENCES}

[1] L. Z. Wu, "A crack in a confocal elliptic piezoelectric inhomogeneity embedded in an infinite piezoelectric medium". International journal of fracture, Vol. 104, No. 1, pp. 1-14 (2000).

[2] Z. Chen, D. Liu, and Z. Yang, "Dynamic stress concentration and scattering of $\mathrm{SH}$-wave by interface elliptic cylindrical cavity". Earthquake Engineering and Engineering Vibration, Vol. 2, No. 2, pp. 299-306 (2003).

[3] J. X. Liu and X. Q. Wang, "Interaction of a screw dislocation with a notch in a piezoelectric bi-material". Archive of Applied Mechanics, Vol. 73, No. 8, pp. 553560 (2004).

[4] D. Liu and $\mathrm{H}$. Lin, "Scattering of SH-waves by an interacting interface linear crack and a circular cavity near biomaterial interface". Acta mechanica sinica, Vol. 20, No. 3, pp. 317-326 (2004).

[5] D. Liu and Z. Chen, "Scattering of SH-wave by cracks originating at an elliptic hole and dynamic stress intensity factor". Applied Mathematics and Mechanics, Vol. 25, No. 9, pp. 1047-1056 (2004).

[6] S. Chen, M. Shen and F. Chen, "Piezoelectric Study on Elliptic Inhomogeneity Problem Using Alternating Technique". Journal of Elasticity, Vol. 81, No. 1, pp. 91-109 (2005).

[7] T. Sasaki, T. Suzuki and K. Hirashima, "Transversely isotropic piezoelectric materials with an arbitrarily shaped boundary". Acta mechanica, Vol. 184, No. 14, pp. 217-230 (2006).

[8] J. H. Uo, P. Liu, Z. X. Lu and T. Y. Qin, "Anti-plane analysis of semi-infinite crack in piezoelectric strip". Applied Mathematics and Mechanics, Vol. 32, pp. 75-82 (2011). 
[9] T. S. Song and A. Hassan, "Dynamic Anti-Plane Analysis for Symmetrically Radial Cracks near a Non-Circular Cavity in piezoelectric bi-materials". Acta Mechanica, Vol. 226, No. 7, pp. 2089-2101 (2015).

[10] T. S. Song and A. Hassan, "Dynamic Performance at Inner Tip of an Interfacial Crack near a Circular Cavity in Piezoelectric Bi-Materials". Applied Mechanics and Materials, Vol. 665, pp. 30-36 (2014).

[11] T. S. Song and A. Hassan, "Dynamic Anti-Plane Characteristics at Outer Tip of an Interfacial Crack near a Circular Cavity in Piezoelectric Bi-Materials". Applied Mechanics and Materials, Vol. 684, pp. 88-93 (2014).

[12] T. S. Song, H. Y. Zheng, H. Li and D. K. Liu, "Dynamic anti-plane characteristic of an infinite piezoelectric medium with non-circular hole". Journal of Harbin Engineering University, Vol. 27, No. 5, pp. 709-713 (2006). (In Chinese).

[13] T. S. Song, D. Li and T. Merhej, "Dynamic stress intensity factor for an interfacial crack on a circular cavity in piezoelectric media". IMECE2010, British Colombia, Canada (2010).

[14] Y.J. Wang and C.F. Gao, "The mode iii cracks originating from the edge of a circular hole in a piezoelectric solid". International Journal of Solids and Structures, Vol. 45, No. 16, pp. 4590-4599 (2008). 


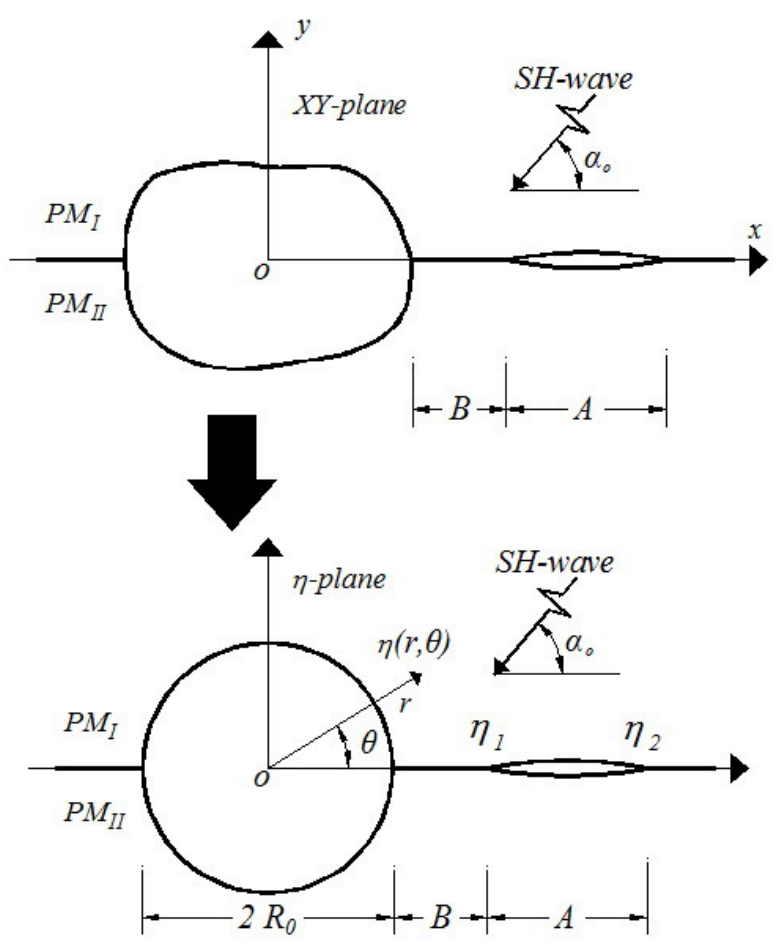

Fig.1. Piezoelectric bi-materials with an interfacial crack near a non-circular cavity and equivalent mapping plane

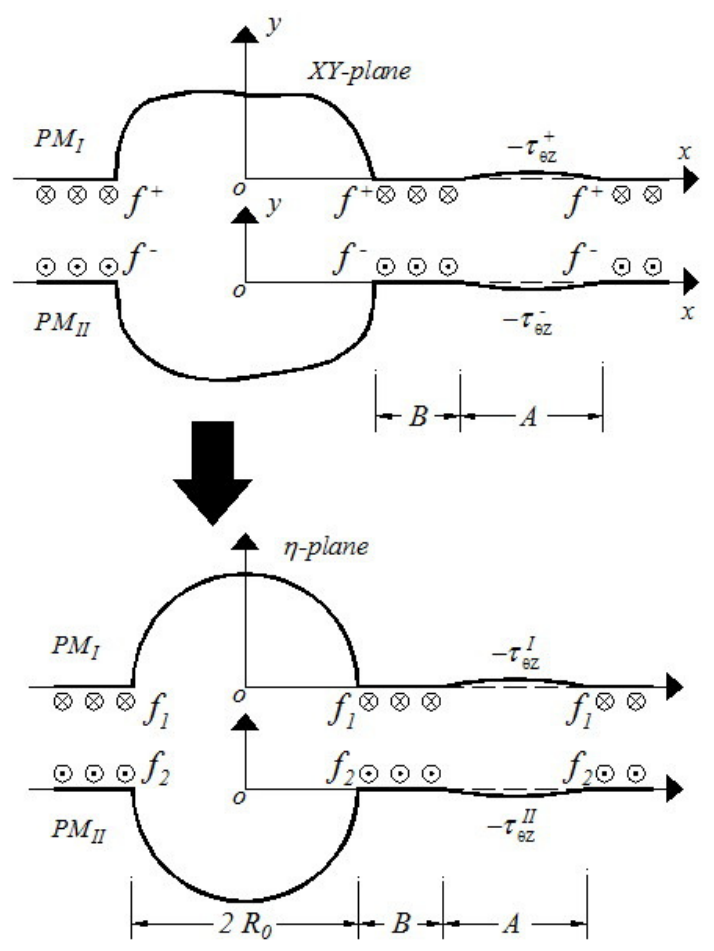

Fig.2. Piezoelectric bi-materials' conjunction with two semi-non-circular notches and equivalent mapping plane

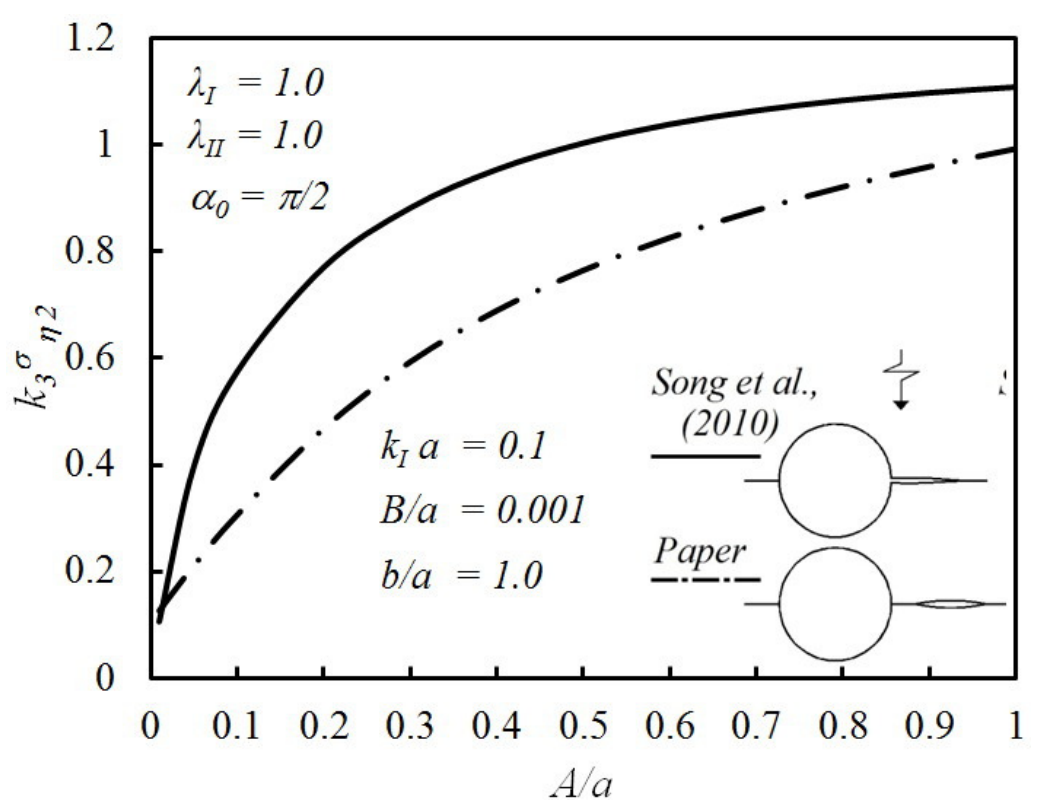

Fig.3. Comparison of DSIFs between different two models under vertical incidence 


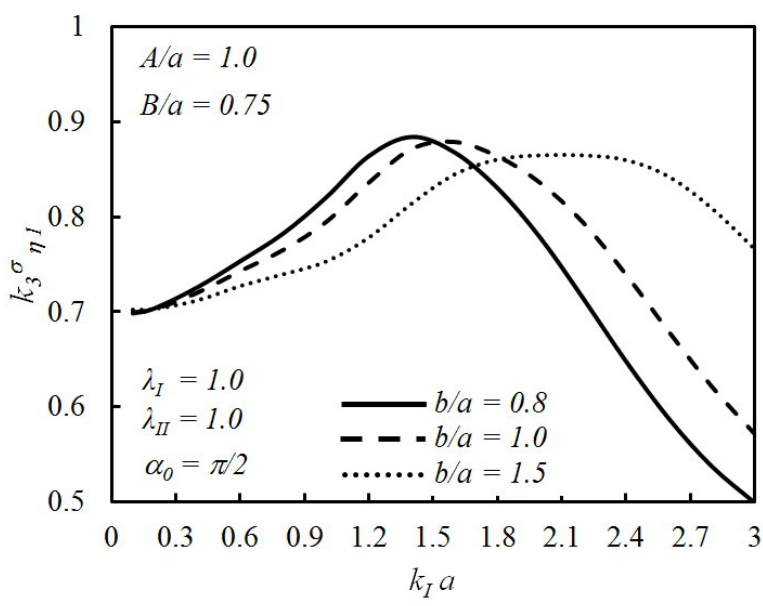

Fig.4. Variations of DSIFs at $\eta_{1}$ with $k_{I} a$ for different semi-axis' percentages of the ellipse

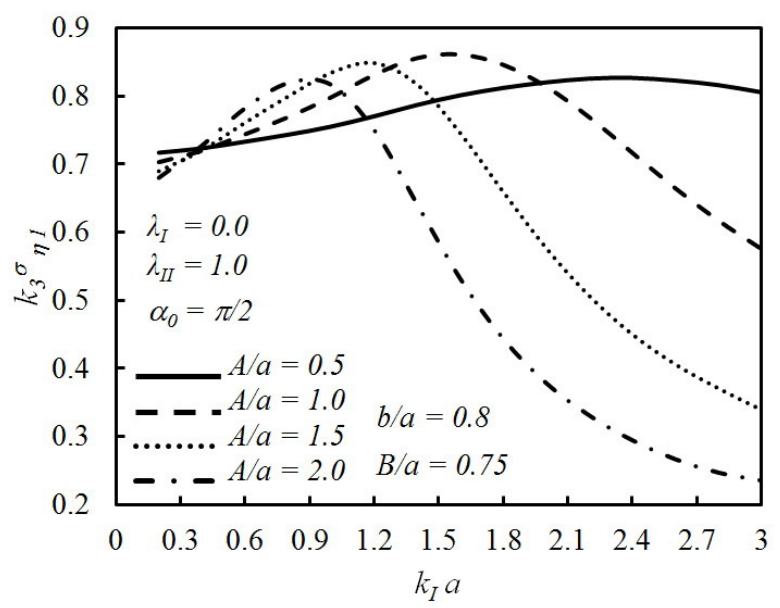

ig.6. Variations of DSIFs at $\eta_{1}$ with $k_{I} a$ for different $A / a$ percentages

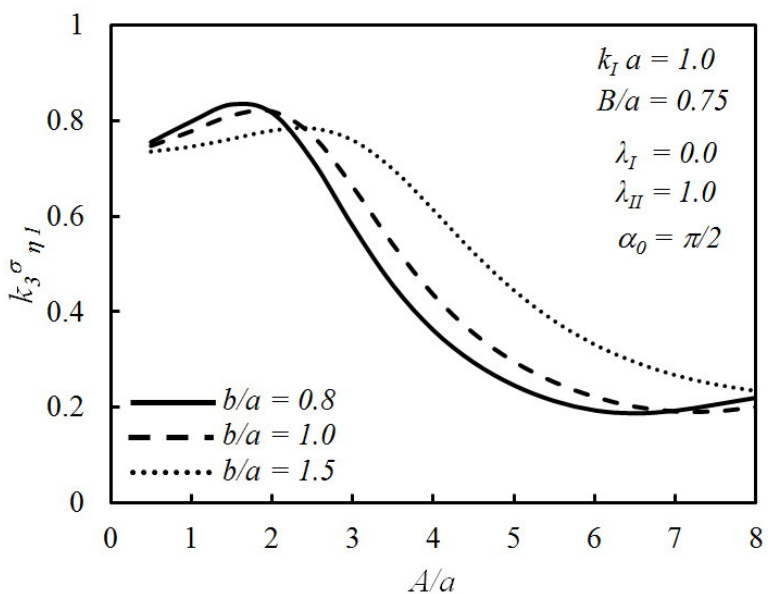

Fig.8. Variations of DSIFs at $\eta_{1}$ with $A / a$ percentage for different semi-axis' percentages

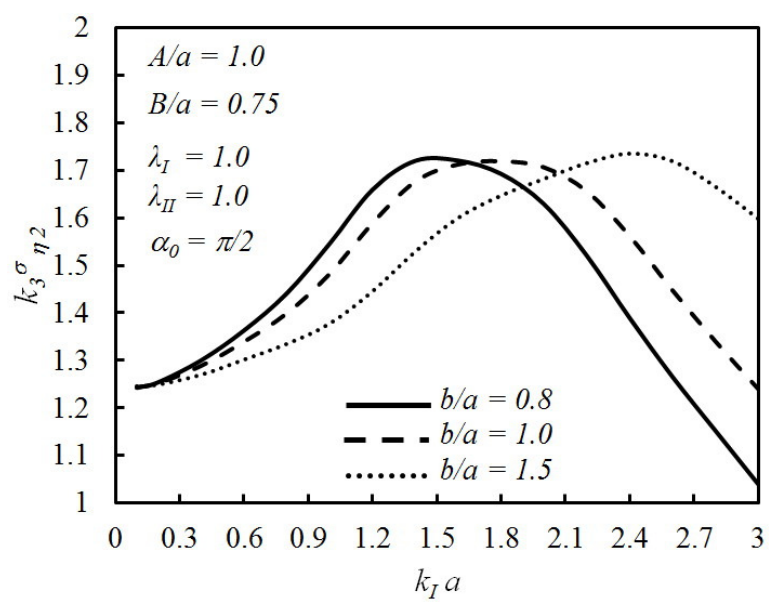

Fig.5. Variations of DSIFs at $\eta_{2}$ with $k_{I} a$ for different semi-axis' percentage of the ellipse

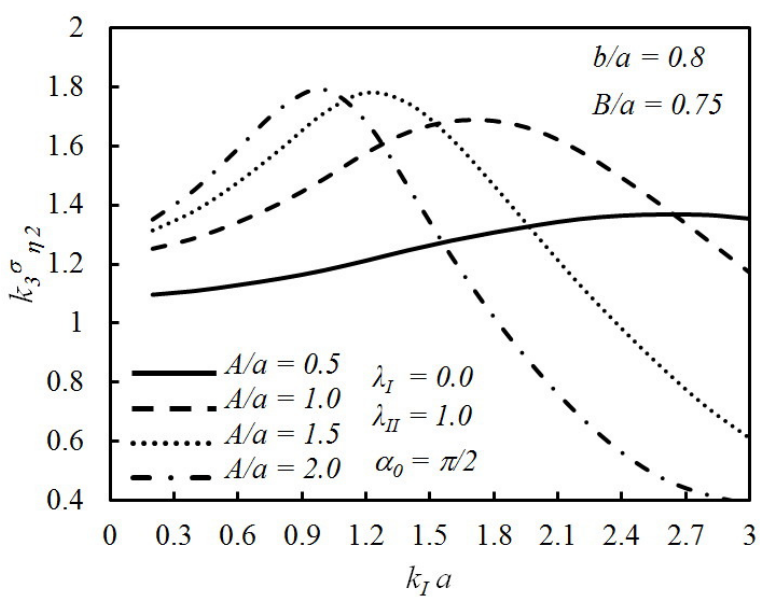

Fig.7. Variations of DSIFs at $\eta_{2}$ with $k_{I} a$ for different $A / a$ percentages

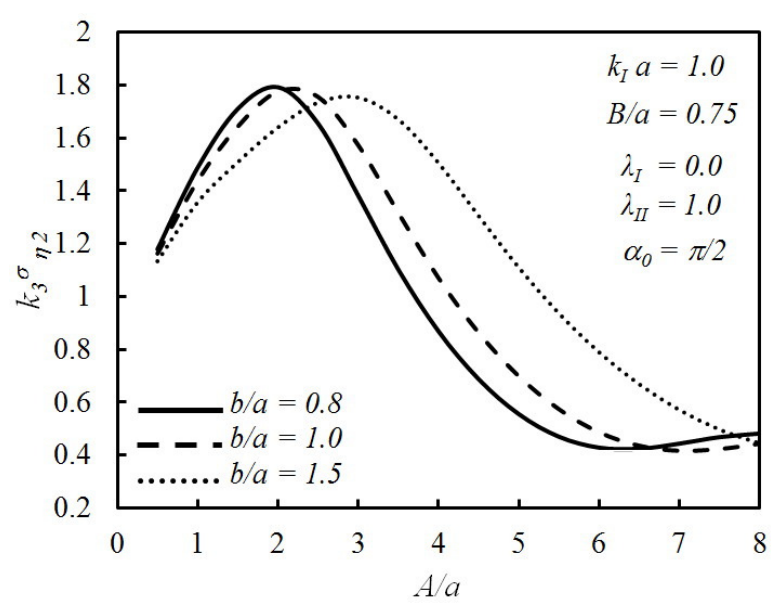

Fig.9. Variations of DSIFs at $\eta_{2}$ with $A / a$ percentage for different semi-axis' percentages 


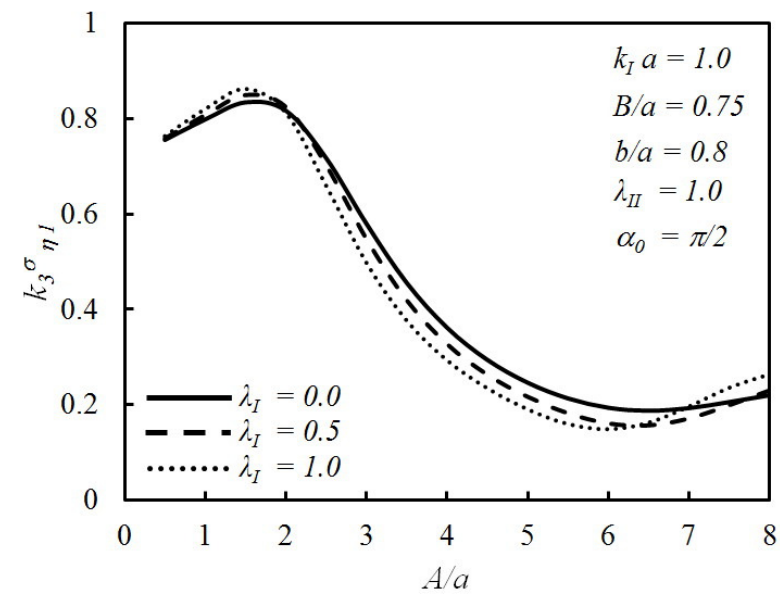

ig.10. Variations of DSIFs at $\eta_{1}$ with $A / a$ Fig.11. Variations of DSIFs at $\eta_{2}$ with $A / a$ percentage for different wave frequencies

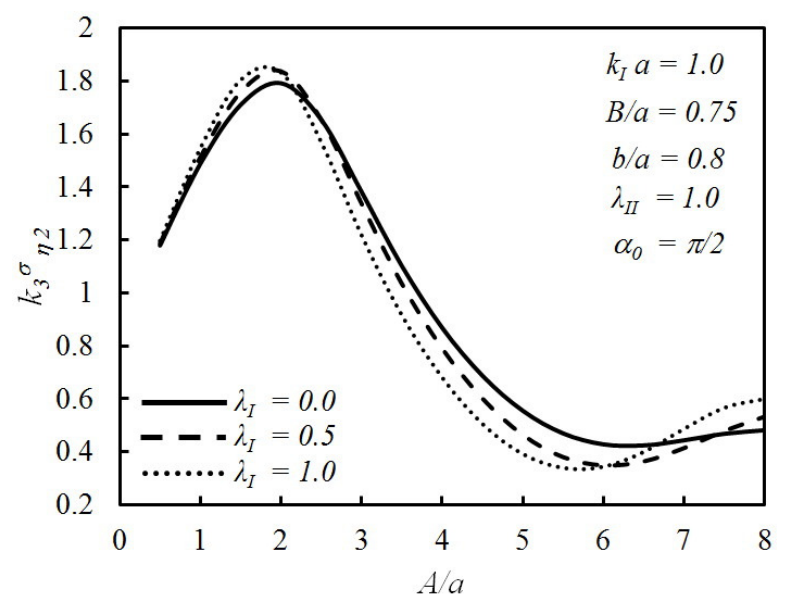

percentage for different wave frequencies 